\title{
Effects of azithromycin on Pseudomonas aeruginosa isolates from catheter-associated urinary tract infection
}

\author{
ZHI-GANG XU ${ }^{1 *}$, YU GAO $^{2 *}$, JIAN-GUO HE ${ }^{1}$, WEI-FENG XU ${ }^{1}$, MEI JIANG ${ }^{1}$ and HUAN-SHENG JIN ${ }^{1}$ \\ ${ }^{1}$ Urologic Institute of Chongqing Red Cross Hospital, Chongqing 400020; ${ }^{2}$ Urologic Institute of Southwest Hospital, \\ Third Military Medical University, Chongqing 400038, P.R. China
}

Received April 14, 2014; Accepted November 13, 2014

DOI: $10.3892 /$ etm.2014.2120

\begin{abstract}
Pseudomonas aeruginosa is a common pathogenic bacterium in urinary tract infections (UTIs), particularly catheter-associated UTIs. The aim of this study was to investigate the effect of azithromycin (AZM) on P. aeruginosa isolated from UTIs. Isolates were identified by biochemical assays and the Vitek system. Antimicrobial susceptibility was determined using the disk diffusion assay. Biofilm formation and adhesion were assayed using a crystal violet staining method. The swimming motility was assayed on agar plates. The elastase activity and rhamnolipid production were determined by the elastin-Congo red method and orcinol reaction, respectively. A total of 32 bacterial isolates were collected from 159 urinary catheters and eight of them were P. aeruginosa isolates. The results showed that the $P$. aeruginosa isolates had stronger biofilm formation capability and the biofilms were thicker than those of P. aeruginosa PAO1. AZM inhibited biofilm formation and adhesion on urinary catheters, and also decreased swimming motility and the production of virulence factors. The results of this study indicated that AZM is potentially a good choice for use in the treatment of UTIs.
\end{abstract}

\section{Introduction}

Urinary tract infections (UTIs) are among the most common infections in both outpatient and inpatient settings. Pseudomonas aeruginosa is a common pathogenic bacteria isolated from UTIs, particularly catheter-associated UTIs $(1,2)$. With the development of medical technology, urinary catheters are applied to greater numbers of people and the time of

Correspondence to: Dr Huan-Sheng Jin, Urologic Institute of Chongqing Red Cross Hospital, No. 1 Jialing 1st Village, Jiangbei, Chongqing 400020, P.R. China

E-mail: huansh_jin@163.com

*Contributed equally

Key words: Pseudomonas aeruginosa, azithromycin, urinary tract infection, biofilm, swimming, virulence factor application is longer. Regarding hospitalized patients, $25 \%$ of patients undergo short-term urinary catheterization ( $<7$ days), which increases the risk of developing an infection. Moreover, the UTI rate could reach $100 \%$ in hospitalized patients with long-term catheterization ( $\geq 30$ days) (3). Once the bacterial biofilm develops, the bacterial cells are able to withstand host immune responses, and they are much less susceptible to antibiotics than their nonattached individual planktonic counterparts (4). Due to multiple resistance mechanisms, the higher resistance is more challenging to the clinician. Therefore, numerous researchers have studied the formation, regulation and resistance of biofilms (5-7).

Macrolides have been shown to have a good effect on $P$. aeruginosa infection in the airway tract. However, they are not a good choice for UTI infections (8), and there is little research concerning the effect of macrolides on UTIs. Therefore, in the present study, $P$. aeruginosa isolates were collected from the urinary catheters of hospitalized patients and the $P$. aeruginosa isolates from the urinary catheters was characterized. In addition, azithromycin (AZM) was selected as a representative macrolide to investigate its effect in treating UTIs.

\section{Materials and methods}

Bacterial strains. Urinary catheters that were applied in hospitalized patients for more than one week were carefully collected under aseptic conditions. The catheters used longer than 7 days were collected and cut open, and a cotton swab was used to scrape the inner wall of possible biofilm infection. The cotton swab was sent immediately to the laboratory for analysis. Isolates were identified by biochemical assays and using the Vitek system (bioMérieux Vitek, Hazelwood, MO, USA). P. aeruginosa PAO1 (American Type Culture Collection, Manassas, VA, USA) was used as the control strain and preserved in the laboratory. The $P$. aeruginosa isolates were examined in the following experiments. The present study was conducted according to the principles of the Declaration of Helsinki (2008), and the experimental protocol was approved by the Ethics Committee of Chongqing Red Cross Hospital (Chongqing, China) (approval number, KY201411). Informed consent was obtained from all patients and their families prior to the collection of urinary catheter samples. 
Antimicrobial susceptibility testing. Antimicrobial susceptibility was determined according to the standards of the Clinical and Laboratory Standards Institute (CLSI, 2011) (9) using the Kirby-Bauer disk diffusion assay on freshly prepared $P$. aeruginosa test medium [Müller-Hinton (MH)]. P. aeruginosa ATCC27853 and Escherichia coli ATCC25922 (American Type Culture Collection) were used as control strains for minimum inhibitory concentration (MIC) testing. The resistance rate was calculated as the number of resistant strains divided by the total number of strains. The susceptible strains included those with susceptibility and medium sensitivity according to the CLSI standards.

Biofilm assay. The static biofilm assay was performed as outlined by Wang et al and Naik et al $(10,11)$. In brief, the $P$. aeruginosa strains were grown overnight in Luria-Bertani (LB) broth and diluted to $1 \times 10^{6} \mathrm{CFU} / \mathrm{ml}$ with fresh $\mathrm{LB}$. The inoculated culture $(150 \mu \mathrm{l})$ was transferred to a 96 -well polystyrene microtiter plate and incubated at $37^{\circ} \mathrm{C}$ for $24 \mathrm{~h}$. The planktonic cells were removed from the wells after incubation and the wells were washed three times with sterile water. This was followed by staining of the wells with $0.1 \%$ crystal violet for $10 \mathrm{~min}$ and washing the unbound stain thrice with sterile water. The cell-bound dye was extracted with $300 \mu 195 \%$ ethanol, and the absorbance of the solution was measured using a Multiskan Spectrum microplate reader at $595 \mathrm{~nm}$ (Thermo Fisher Scientific, Vantaa, Finland).

Adhesion on urinary catheters. The adhesion on urinary catheters was assayed using the standard crystal violet staining method with a few revisions (12). In brief, a 3-cm urinary catheter was placed into a 6 -well board with $4.5 \mathrm{ml} \mathrm{LB}$ broth, and $50 \mu \mathrm{l}$ bacterial culture $\left(1 \times 10^{6} \mathrm{CFU} / \mathrm{ml}\right)$ was added to the well. After incubation for $6 \mathrm{~h}$, unattached cells were removed with sterile water, and attached cells were stained with $1 \%$ crystal violet for $20 \mathrm{~min}$. The dye bound to the adherent cells was then solubilized with ethanol-acetone $(75: 15, \mathrm{v} / \mathrm{v})$ and the optical density of the solution was measured at $570 \mathrm{~nm}$.

Swimming motility. The swimming motility was assayed using the method of Bala et al (13). Bacterial strains were incubated at $37^{\circ} \mathrm{C}$ overnight. Swimming plates containing $1 \%$ tryptone, $0.5 \% \mathrm{NaCl}$ and $0.3 \%$ agar were prepared for the assay. The plates with and without 1/4 MIC AZM were point-inoculated with a sterile toothpick and incubated at $37^{\circ} \mathrm{C}$ for $24 \mathrm{~h}$. The zone diameter was measured to assess the swimming motility.

\section{Quantitative analysis of virulence factor production}

Determination of elastase activity. Elastase activity was determined with elastin-Congo red (ECR) and the steps were as follows: $P$. aeruginosa strains were inoculated onto $\mathrm{MH}$ agar plates, and incubated at $37^{\circ} \mathrm{C}$ for $18 \mathrm{~h}$. A single colony was transferred into $2 \mathrm{ml}$ peptone tryptic soy broth (PTSB) medium, and then the bacterial cultures were transferred into $18 \mathrm{ml}$ PTSB medium with and without 1/4 MIC AZM once the $\mathrm{OD}_{540 \mathrm{~nm}}$ reached 0.5 . After incubation for $16 \mathrm{~h}$ at $37^{\circ} \mathrm{C}$ with shaking at $250 \mathrm{rpm}$, the cultures were centrifuged at $12,100 \mathrm{x} \mathrm{g}$ for $15 \mathrm{~min}$ at $4^{\circ} \mathrm{C}$ and the supernatant was filtered with $0.45 \mu \mathrm{m}$ syringe filter. Then, $1 \mathrm{ml}$ ECR reaction buffer
(ECR 20 mg, $0.1 \mathrm{M}$ Tris-HCl/1 mM CaCl 2 , pH 7.2) was added to $1 \mathrm{ml}$ filtered supernatant. When the mixture had been incubated at $37^{\circ} \mathrm{C}$ for $18 \mathrm{~h}$ with shaking at $250 \mathrm{rpm}, 0.1 \mathrm{ml} 0.12 \mathrm{M}$ EDTA was added to stop the reaction. The reactant was placed on ice and the insoluble ECR was removed by centrifugation at $3,000 \mathrm{x}, 4^{\circ} \mathrm{C}$. The elastase activity was determined at $495 \mathrm{~nm}$. Three samples of each type were examined and the experiment was repeated three times.

Determination of rhamnolipids. The concentration of rhamnolipid was determined spectrophotometrically by the orcinol reaction using rhamnose as a standard. The orcinol reagent $[0.19 \%$ orcinol in $53 \%$ (v/v) sulfuric acid] was prepared immediately prior to use. The reaction mixture, composed of $100 \mu \mathrm{l}$ sample $(0,50,100,200$ and $300 \mu \mathrm{g} / \mathrm{ml})$ and $900 \mu \mathrm{l}$ reagent, was well stirred, warmed for $30 \mathrm{~min}$ at $80^{\circ} \mathrm{C}$, and then kept for $15 \mathrm{~min}$ at room temperature. The absorbance was measured at $421 \mathrm{~nm}$. A standard curve was constructed according to the absorbance and concentration of rhamnose.

$P$. aeruginosa strains were grown on LB agar plates at $37^{\circ} \mathrm{C}$ for $18 \mathrm{~h}$. A single colony was transferred into LB broth and incubated overnight at $37^{\circ} \mathrm{C}$ with shaking. Then, the $\mathrm{OD}_{600 \mathrm{~nm}}$ of the bacterial culture was adjusted to 0.05 with proteose peptone glucose ammonium salts (PPGAS) medium $\left(0.02 \mathrm{M} \mathrm{NH}_{4} \mathrm{Cl}, 0.02 \mathrm{M} \mathrm{KCl}, 0.12 \mathrm{M}\right.$ Tris-HCl, $0.0016 \mathrm{M}$ $\mathrm{MgSO}_{4}, 1 \%$ peptone and $0.5 \%$ glucose). Bacterial cultures (20 ml) with and without 1/4 MIC AZM were incubated for $48-72 \mathrm{~h}$ at $37^{\circ} \mathrm{C}$ with shaking at $200 \mathrm{rpm}$. Rhamnolipids were purified by first separating the cells from the supernatant by centrifugation at $6,000 \mathrm{x}$ f for $10 \mathrm{~min}$. The supernatant was then acidified using $12 \mathrm{M}$ hydrochloric acid to $\mathrm{pH} 2.0$, and the precipitated rhamnolipids were collected by centrifugation at $12,100 \mathrm{x}$ g for $5 \mathrm{~min}$. Rhamnolipids were extracted twice with ethyl acetate, which was then evaporated away leaving behind relatively pure rhamnolipids. The rhamnolipids

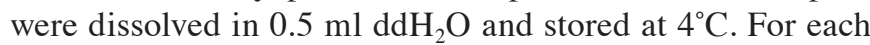
sample, $100 \mu \mathrm{l}$ rhamnolipids were determined spectrophotometrically using this method. The content of rhamnose was calculated on the basis of the standard curve. The concentration of rhamnolipid was calculated based on the assumption that $1 \mu \mathrm{g}$ rhamnose corresponds to $2.5 \mu \mathrm{g}$ rhamnolipid.

\section{Results}

Bacterial strains and susceptibility. A total of 159 urinary catheters were collected and 32 showed positive bacterial cultures. Six urinary catheters had more than two kinds of bacteria. Eight $P$. aeruginosa isolates were collected from the urinary catheters. The resistance rates of the eight $P$. aeruginosa isolates to amikacin, ciprofloxacin, levofloxacin, minocycline, ceftazidime, cefotaxime, piperacillin, meropenem, netilmicin, tetracycline and cefepime were 87.5, 87.5, 75.0, 62.5, 87.5, 75.0, 100.0, 62.5, 75.0, 87.5 and 75.0\%, respectively. The 1/4 MIC values of AZM on the isolates of P. aeruginosa are presented in Table I. The 1/4 MICs ranged from 32 to $256 \mu \mathrm{g} / \mathrm{ml}$.

Biofilm formation and adhesion. The effects of AZM on the biofilm formation and adhesion of the P. aeruginosa isolates are shown in Table I. The strains from urinary catheters had stronger biofilm formation capability, and the biofilms of eight 
Table I. Effect of 1/4 MIC azithromycin on the biofilm formation and adhesion of P. aeruginosa.

\begin{tabular}{lccccccccc}
\hline Variable & PAO1 & PA1 & PA2 & PA3 & PA4 & PA5 & PA6 & PA7 & PA8 \\
\hline 1/4MIC & 64 & 128 & 64 & 64 & 128 & 64 & 256 & 32 & 64 \\
OD $_{\text {biofilm }}$ & 0.46 & 0.33 & 0.48 & 0.32 & 0.66 & 0.27 & 0.68 & 0.37 & 0.39 \\
OD $_{\text {adhesion }}$ & 0.43 & 0.52 & 0.47 & 0.44 & 0.36 & 0.36 & 0.31 & 0.61 & 0.47 \\
\hline
\end{tabular}

MIC, minimum inhibitory concentration; OD, optical density. $\mathrm{OD}_{\text {bifilm }}$ and $\mathrm{OD}_{\text {adhesion }}$ indicate the ratios of $\mathrm{OD}$ values between the $1 / 4 \mathrm{MIC}$ AZM group and the control group. PAO1, control strain; PA1-8, P. aeruginosa clinical isolates.
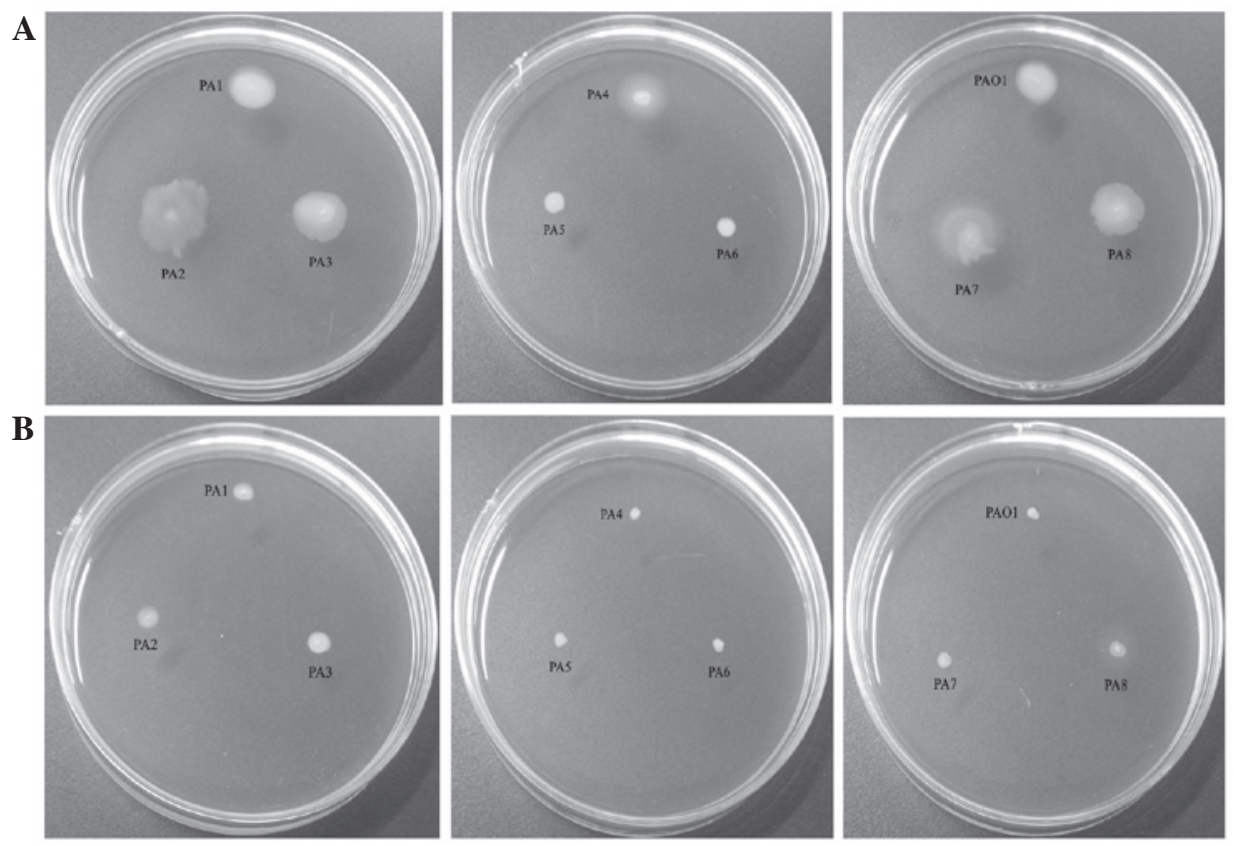

Figure 1. Demonstration of the swimming motility of standard strain PAO1 and P. aeruginosa clinical isolates PA1-PA8 (A) in the absence (control group) and (B) presence (tested group) of azithromycin.

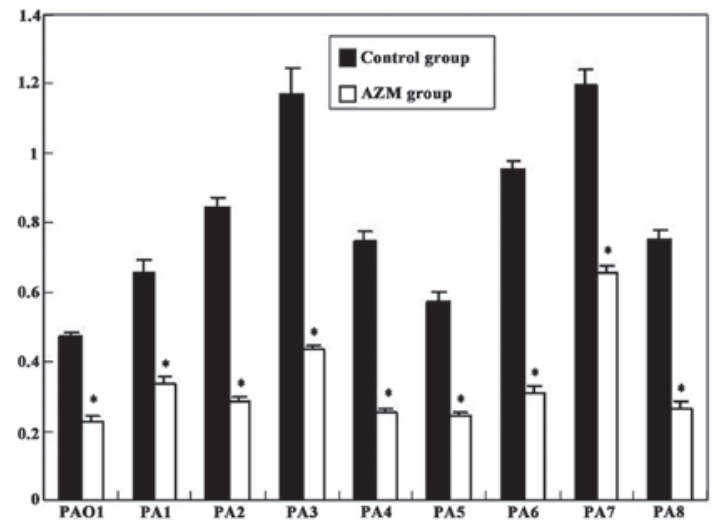

Figure 2. Elastase activity of P. aeruginosa standard strain PAO1 and isolates PA1-PA8. " $\mathrm{P}<0.01$, compared with the control group.

isolates were thicker than those of P. aeruginosa PAO1 (data not shown) The 1/4 MIC AZM may reduce the biofilm formation capability and the adhesion to urinary catheters.

Swimming motility. The results of the assay investigating the effects of AZM on swimming motility are presented in

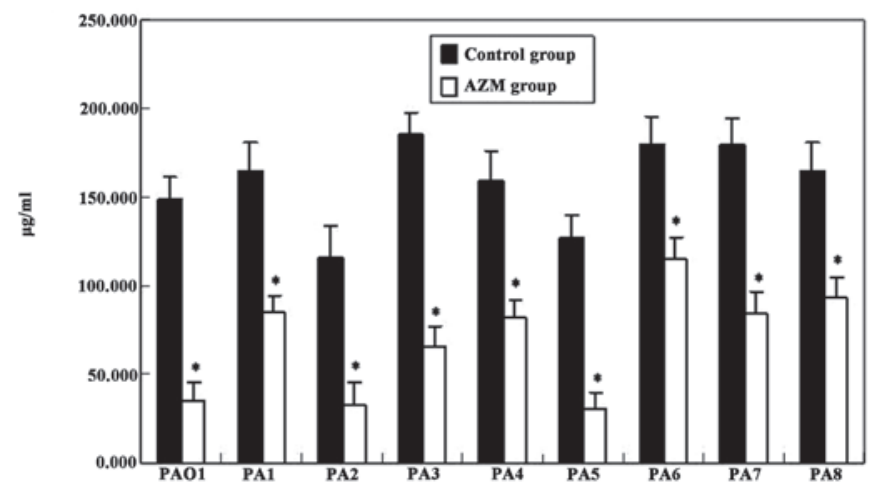

Figure 3. Rhamnolipid production by of P. aeruginosa standard strain PAO1 and isolates PA1-PA8. ${ }^{*} \mathrm{P}<0.01$, compared with the control group.

Fig. 1. It was observed that the presence of AZM significantly reduced the motility of all $P$. aeruginosa strains.

Virulence factors. The elastase activity of all P. aeruginosa clinical isolates was higher than that of PAO1 in the control and AZM groups, respectively (Fig. 2). The production of 
rhamnolipids by the $P$. aeruginosa clinical isolates was higher than that by PAO1, with the exception of isolates PA2 and PA5 (Fig. 3). Moreover, the presence of AZM in the culture medium greatly decreased the expression of these virulence factors ( $\mathrm{P}<0.01$; Figs. 2 and 3).

\section{Discussion}

With the development of medical technology, urinary catheterization is increasingly common in hospitalized patients, which is a risk factor of bacteriuria and symptomatic UTI (14). In the present study, the rate of isolation of positive strains was $20 \%$, and the $P$. aeruginosa-positive rate was 5\%. Among the eight obtained isolates, the biofilms were thicker than those of $P$. aeruginosa $\mathrm{PAO1}$, which suggested that the strains isolated from the urinary catheters had stronger biofilm formation capability. Therefore, for $P$. aeruginosa in UTI, the possibility of biofilm formation during therapy should be considered. Swimming motility is a significant etiological factor. In the presence of 1/4 MIC AZM, the swimming motility was depressed greatly. Moreover, AZM not only decreased biofilm formation capability, but also decreased the adhesion to urinary catheters, which is similar to the findings of previous studies $(13,15)$.

P.aeruginosa elastase (PE) is a $39.5-\mathrm{kDa}$ metalloproteinase and one of the strongest virulence factors of $P$. aeruginosa, which can degrade the elastin of human matrix proteins including laminin and collagen types III and IV (16-18). PE has also been found to be an immunosuppressive factor $(19,20)$. In biofilm formation, rhamnolipids have important actions, which are involved in modulating the swarming and colonization of incipient biofilm forming (21). The inhibitory effect of AZM on the production of rhamnolipids was less significant than its inhibitory effect on elastin. However, the rhamnolipid levels were markedly decreased. The results demonstrated that AZM decreased the elastase activity greatly, indicating that AZM might relieve the pathogenicity of $P$. aeruginosa from UTIs.

AZM has been shown to exert a good therapeutic effect on cystic fibrosis (22). However, to the best of our knowledge, there are few reports concerning the use of AZM in the treatment of UTIs. Administration of prophylactic antibiotics following catheter application decreases UTI rates (23). However, prophylactic antibiotics are usually trimethoprim-sulfamethoxazole and quinolones (23). Macrolides are not a good choice for UTIs according to the clinical application principles of antibiotics of China (8). As AZM is a new macrolide antibiotic, it is inferred from the present study results that macrolides may be a good choice in the treatment of UTIs involving P. aeruginosa. However, there are many factors affecting the clinical treatment effect, such as the immunity condition of patients, the original disease and resistance of $P$. aeruginosa. Therefore, it is necessary to conduct further clinical studies.

\section{References}

1. Ronald A: The etiology of urinary tract infection: traditional and emerging pathogens. Am J Med 113 (Suppl 1A): 14S-19S, 2002.

2. Jarvis WR and Martone WJ: Predominant pathogens in hospital infections. J Antimicrob Chemother 29 (Suppl A): 19-24, 1992.
3. Stickler DJ: Bacterial biofilms and the encrustation of urethral catheters. Biofouling 9: 293-305, 1996.

4. Nickel JC, Ruseska I, Wright JB and Costerton JW: Tobramycin resistance of Pseudomonas aeruginosa cells growing as a biofilm on urinary catheter material. Antimicrob Agents Chemother 27: 619-624, 1985

5. Cole SJ, Records AR, Orr MW, Linden SB and Lee VT: Catheterassociated urinary tract infection by Pseudomonas aeruginosa is mediated by exopolysaccharide-independent biofilms. Infect Immun 82: 2048-2058, 2014.

6. Tielen P, Rosin N, Meyer AK, et al: Regulatory and metabolic networks for the adaptation of Pseudomonas aeruginosa biofilms to urinary tract-like conditions. PLoS One 8: e71845, 2013.

7. Narten M, Rosin N, Schobert M and Tielen P: Susceptibility of Pseudomonas aeruginosa urinary tract isolates and influence of urinary tract conditions on antibiotic tolerance. Curr Microbiol 64: 7-16, 2012.

8. Ministry of Health, Administration of Traditional Chinese Medicine and General Logistics Department of Health: Guidelines for Clinical Use of Antibiotics. Chinese Medical Association, Beijing, China, 2004.

9. Clinical Laboratory Standards Institute: Performance standards for antimicrobial susceptibility testing; 21st informational supplement. M100-S21, Vol 31. Clinical Laboratory Standards Institute, Wayne, PA, USA, 2011.

10. Wang Q, Sun FJ, Liu Y, Xiong LR, Xie LL and Xia PY: Enhancement of biofilm formation by subinhibitory concentrations of macrolides in icaADBC-positive and -negative clinical isolates of Staphylococcus epidermidis. Antimicrob Agents Chemother 54: 2707-2711, 2010.

11. Naik DN, Wahidullah S and Meena RM: Attenuation of Pseudomonas aeruginosa virulence by marine invertebrate-derived Streptomyces sp. Lett Appl Microbiol 56: 197-207, 2013.

12. Tomaras AP, Dorsey CW, Edelmann RE and Actis LA: Attachment to and biofilm formation on abiotic surfaces by Acinetobacter baumannii: involvement of a novel chaperone-usher pili assembly system. Microbiology 149: 3473-3484, 2003.

13. Bala A, Kumar R and Harjai K: Inhibition of quorum sensing in Pseudomonas aeruginosa by azithromycin and its effectiveness in urinary tract infections. J Med Microbiol 60: 300-306, 2011.

14. Saint S and Lipsky BA: Preventing catheter-related bacteriuria: should we? Can we? How? Arch Intern Med 159: 800-808, 1999.

15. Vranes J: Effect of subminimal inhibitory concentrations of azithromycin on adherence of Pseudomonas aeruginosa to polystyrene. J Chemother 12: 280-285, 2000.

16. Wretlind B and Pavlovskis OR: Pseudomonas aeruginosa elastase and its role in pseudomonas infections. Rev Infect Dis 5 (Suppl 5): S998-S1004, 1983.

17. Saulnier JM, Curtil FM, Duclos MC and Wallach JM: Elastolytic activity of Pseudomonas aeruginosa elastase. Biochim Biophys Acta 995: 285-290, 1989.

18. Bejarano PA, Langeveld JP, Hudson BG and Noelken ME: Degradation of basement membranes by Pseudomonas aeruginosa elastase. Infect Immun 57: 3783-3787, 1989.

19. Kharazmi A, Döring G, Høiby N and Valerius NH: Interaction of Pseudomonas aeruginosa alkaline protease and elastase with human polymorphonuclear leukocytes in vitro. Infect Immun 43: $161-165,1984$.

20. Jacquot J, Tournier JM and Puchelle E: In vitro evidence that human airway lysozyme is cleaved and inactivated by Pseudomonas aeruginosa elastase and not by human leukocyte elastase. Infect Immun 47: 555-560, 1985.

21. Caiazza NC,Shanks RMand O'Toole GA: Rhamnolipids modulate swarming motility patterns of Pseudomonas aeruginosa. J Bacteriol 187: 7351-7361, 2005.

22. van Delden C, Köhler T, Brunner-Ferber F, François B, Carlet J, Pechère JC: Azithromycin to prevent Pseudomonas aeruginosa ventilator-associated pneumonia by inhibition of quorum sensing: a randomized controlled trial. Intensive Care Med 38: 1118-1125, 2012.

23. Marschall J, Carpenter CR, Fowler S, Trautner BW; CDC Prevevention Epicenters Program: Antibiotic prophylaxis for urinary tract infections after removal of urinary catheter: meta-analysis. BMJ 346: f3147, 2013. 